\title{
The Impulse Analysis of the Regular Singular System via Kronecker Indices
}

\author{
Zhenghong Jin ${ }^{1,2}$, Qingling Zhang ${ }^{1,2, *}$ and Yi Zhang ${ }^{2,3}$ \\ ${ }^{1}$ Institute of Systems Science, Northeastern University, Shenyang, 110819, China \\ ${ }^{2}$ State Key Laboratory of Synthetical Automation for Process Industries, Northeastern University, Shenyang, 110819, China \\ ${ }^{3}$ School of Science, Shenyang University of Technology, Shenyang, 110870, China \\ ${ }^{*}$ Corresponding author
}

\begin{abstract}
This The problem of impulse analysis in the regular singular system is considered by using the Kronecker indices in this paper. Firstly, the singular system is divided into two cases: 1 . The regular linear singular system. 2 . The uniformly regular nonlinear singular system. Then, the two theorems that the singular is impulsive if and only if the Kronecker indices are more than one are given. Finally, two numerical simulations are carried out to show the consistency with theoretical analysis and illustrate the effectiveness of the algorithm.
\end{abstract}

Keywords_-impulses; regularity; the Kronecker indices

\section{INTRODUCTION}

It is well known that the differential algebraic system has strong applied background and it has been followed with interest extensively for a long time ${ }^{[1-5]}$. Some properties of the singular system were similar to the normal system, such as stability, controllability, observability and detectability. These basic features constituted the internal structural features of the singular system. However, an important difference between a singular system and a normal system is that the singular system is impulsive. Therefore, the impulse is an important feature of the singular system and it has been studied in the literatures ${ }^{[6,7]}$ Potter ${ }^{[8]}$ has extended the standard linear technique of impulse response function analysis to the nonlinear case by defining a generalized impulse response function. But the existence of impulse can be judged based on solution of the singular system in the literature. It can be seen that the solutions of singular systems contain not only exponential terms similar to normal systems, but also the impulse terms and input derivatives. The solution of this form is called the distribution solution in the sense of generalized function. After that, several sufficient conditions for the impulse analysis of the system are obtained. So far, it is not convenient and systematic to judge the existence of the impulse in the singular system. In this paper, we give some sufficient and necessary conditions to determine the existence of the impulse by converting the system into the Kronecker form or the generalized Kronecker form.

On the other hand, regularity is also a characteristic of a singular system and the normal system does not have this property. When given an admissible initial condition, the sufficient and necessary conditions for the existence of the unique solution is that the singular system is regular. Thus, in order to ensure the existence and uniqueness of the solution of singular system, regularity is the most basic requirement for the singular system. In the literature ${ }^{[9]}$, Aldybekov considered the asymptotic solutions of singular systems that defined by generalized exponents from the set of differential equations and the regularity of the singular system was fully analyzed. In the literatures ${ }^{[10-13]}$, Bunse et al. surveys numerical techniques for the regularization of the singular system by various methods such as proportional and derivative feedback.

It has been shown that a singular linear system is impulsive if and only if its indices of Kronecker canonical equivalence form reduce ${ }^{[14]}$. This standard decomposition form performs a very fundamental role in the literatures ${ }^{[6,7]}$. It shows that the Kronecker canonical form (which is a canonical decomposition for pairs of matrices) is the representation of a linear relation in a finite dimensional space and provides a new geometric view upon the Kronecker canonical form for singular system ${ }^{[15]}$. The Kronecker canonical form is an important form of matrix pair and a standard type of singular system. In the literature ${ }^{[16]}$, it uses the Kronecker canonical form to determine whether the singularity induced bifurcation system has an impulse and an important theorem is obtained that if the Kronecker indices of system changes from 1 to 2, the system is impulsive. In the literature ${ }^{[17]}$, it describes the possible numbers of Kronecker indices of the pencils $s E-A$. In this paper, we mainly study the relationship between the impulse and the Kronecker form for four kinds of different singular system. We use the Kronecker form indices for the regular linear and nonlinear singular system.

The organization structure of the paper is as follows. We give a short but self-contained introduction to the theory of linear and nonlinear singular system in this paper in Section II. In Section III, the two classes of singular systems, the regular linear singular system, the uniformly regular nonlinear singular system are given. Then, we give two theorems on the existence of system impulses by using the Kronecker indices. In Section IV, two numerical examples are presented to illustrate the utility of our theoretical results. Section V concludes the paper.

\section{A. Notations:}

Hereafter, the $A^{T}$ denotes transposition of vector or matrix $A$; The symbol $R^{n}$ represents the n-dimensional vector space; $\operatorname{det}(A)$ represents the determinant of matrix $A$; $\operatorname{deg}(\cdot)$ represents degree of polynomial. 


\section{PRELIMINARIES AND PROBLEM FORMULATION}

In this section, we give the description of problem that it finds the relationship between impulse and Kronecker indices in a singular system. We break this problem into two subproblems according to the difference of singular system: 1 . The regular linear singular system. 2. The uniformly regular nonlinear singular system.

Consider the following linear singular system

$$
E \dot{x}(t)=A x(t)+B u(t)
$$

where $x(t) \in R^{n}$ is the state vector and $u(t) \in R^{m}$ is the input vector. $E \in R^{n \times n}$ is a singular matrix, $\operatorname{rank}(E)=r(\leq n), A \in R^{n \times n}$ and $B \in R^{n \times m}$ are the constant matrices.

Definition 1. The system (1) is called regular if there exists a constant scalar $s_{0} \in \mathbb{C}$ and satisfied

$$
\operatorname{det}\left(s_{0} E-A\right) \neq 0
$$

In this case, we also say that the matrix pair $(E, A)$ is regular.

\section{Definition 2 (Kronecker indices)}

A regular matrix pencil $(E, A)$ admits nonsingular matrices $P, Q \in \mathbb{R}^{n \times n}$ such that

$$
P E Q=\left[\begin{array}{cc}
I_{r} & 0 \\
0 & N
\end{array}\right], P A Q=\left[\begin{array}{cc}
W & 0 \\
0 & I_{n-r}
\end{array}\right]
$$

where $W \in \mathbb{R}^{r \times r}$ for some $r \leq n$ and $N \in \mathbb{R}^{(n-r) \times(n-r)}$ is a nilpotent matrix with index $k \leq n-r$, that is, $N^{k}=0$ and $N^{k-1} \neq 0$. The nilpotency index $k$ is called the Kronecker indices.

Lemma $\mathbf{1}^{[6]}$. The regular linear singular system (1) is impulse-free if and only if one of the following conditions holds:

1. The nilpotent matrix $N=0$;

2. $\operatorname{deg}(\operatorname{det}(s E-A))=\operatorname{rank}(E)$

Next, we consider a nonlinear singular system.

$$
E \dot{x}(t)=f(x(t), u(t), t)
$$

where $f$ is a n-dimensional nonlinear function with $x(t), u(t)$ and $t$.

For the nonlinear singular system (2), the T-S fuzzy singular system is given

\section{Model Rule $i$ :}

IF $\mathrm{z}_{1}(\mathrm{t})$ is $M_{i 1}$ and $\cdots$ and $\mathrm{z}_{k}(\mathrm{t})$ is $M_{i k}$

$$
\text { THEN } E \dot{x}(t)=A_{i} x(t)+B_{i} u(t), i=1,2, \cdots, r
$$

where $\mathrm{M}_{i j}$ is the fuzzy set and $r$ is the number of model rules. $z_{1}(t), \cdots, z_{k}(t)$ are the premise variables and they are function of the state variables. Let $z(t)=\left[\begin{array}{lll}z_{1}(t) & \cdots & z_{k}(t)\end{array}\right]^{T}$. The T-S fuzzy model can be given by using product inference machine, the center-average weighted defuzzifer and gaussian membership functions

$$
E \dot{x}(t)=\sum_{i=1}^{r} h_{i}(z(t))\left[A_{i} x(t)+B_{i} u(t)\right]
$$

where $\beta_{i}(z(t))=\prod_{j=1}^{k} M_{i j}\left(z_{j}(t)\right), \quad{ }_{i}(z(t))=\frac{\beta_{i}(z(t))}{\sum_{i=1}^{r} \beta_{i}(z(t))} \geq 0$, $\sum_{i=1}^{r} h_{i}(z(t))=1, \quad M_{i j}(\cdot)$ is the grade of membership of $z_{i}(t)$ in fuzzy set $M_{i j}$ and $h_{i}(z(t))$ is the normalized weight of each fuzzy rules.

Remark 1. The regularity of nonlinear singular system is represented the regularity of the T-S fuzzy model of the nonlinear singular system. If the T-S fuzzy model is uniformly regular, the nonlinear singular system is called uniformly regular.

Lemma $2^{[7]}$. The uniformly regular nonlinear singular system (2) or T-S fuzzy singular system (3) is impulse-free if and only if the following condition holds:

$$
\operatorname{deg}\left(\operatorname{det}\left(s E-\sum_{i=1}^{r} h_{i}(z(t)) A_{i}\right)\right)=\operatorname{rank}(E), \forall t
$$

\section{MAIN RESULTS}

In this section, we mainly analyze the relationship between the impulse and the Kronecker indices for two types of singular systems.

\section{A. The Regular Linear Singular System}

The Kronecker form of a regular linear singular system (1) can be given. That is, there exist two nonsingular matrices $P, Q \in \mathbb{R}^{n \times n}$ such that

$$
P E Q=\left[\begin{array}{cc}
I_{r} & 0 \\
0 & N
\end{array}\right], P A Q=\left[\begin{array}{cc}
A_{1} & 0 \\
0 & I_{n-r}
\end{array}\right]
$$

where $A_{1} \in \mathbb{R}^{r \times r}$.

Theorem 1. For the regular linear singular system (1), it is impulsive if and only if the Kronecker indices $k$ is more than one, that is $k>1$. 
Proof: The regular linear singular system (1) is impulsive, so the nilpotent matrix $N \neq 0$ of the Kronecker form (4) of system (1) based on the Lemma 1. Then the $N^{k}=0$ if and only if $k>1$.

\section{B. The Uniformly Regular Nonlinear Singular System}

For a uniformly regular nonlinear singular system as form equation (2), it is known that the T-S fuzzy singular system (2) as form system (3) and system (2) are equivalent about some properties of the system, such as stability, regularity and impulsiveness. So, we can analyze the relationship between the impulse and the Kronecker indices for the uniformly regular nonlinear singular system (2) by using the T-S fuzzy singular system (3). When the nonlinear singular system (2) is uniformly regular, the T-S fuzzy singular system (3) is also uniformly regular, that is, $\operatorname{det}\left(s E-\sum_{i=1}^{r} h_{i}(z(t)) A_{i}\right) \neq 0, \forall t$.

The Kronecker form of the T-S fuzzy singular system (3) can be given. Firstly, let $A=\sum_{i=1}^{r} h_{i}(z(t)) A_{i}$ and $\operatorname{det}(s E-A) \neq 0, \forall t$, so there exist two nonsingular matrices $P, Q \in \mathbb{R}^{n \times n}$ and satisfy

$$
P E Q=\left[\begin{array}{cc}
I_{r} & 0 \\
0 & N
\end{array}\right], P A Q=\left[\begin{array}{cc}
M & 0 \\
0 & I_{n-r}
\end{array}\right]
$$

where $M \in \mathbb{R}^{r \times r}$ and the matrix $N$ is a nilpotent matrix and the nilpotency index $k$ is called the Kronecker indices of the T-S fuzzy singular system (3). Because the matrix $A$ is timevarying, the matrices $M$ and $N$ may be time-varying.

Remark 2. In this paper, the nonlinear singular systems are sector nonlinear system and can be transformed into T-S fuzzy singular systems. The non sector nonlinear singular systems are not within the scope of research.

Theorem 2. For the uniformly regular nonlinear singular system (2), it is impulsive if and only if the Kronecker indices $k$ of the T-S fuzzy singular system (3) is more than one, that is $k>1$.

Proof: Because the nonlinear singular system (2) is uniformly regular, the T-S fuzzy singular system (3) is uniformly regular. According to the Lemma 2, the T-S fuzzy singular system (3) is impulsive if and only if $\operatorname{deg}(\operatorname{det}(s E-A)) \neq \operatorname{rank}(E), \exists t$. Therefore, the matrix $N$ of Kronecker form (4) is not equal to 0 . That is, $N^{k}=0$ if and only if $k>1$. So, the nonlinear singular system (2) is impulsive if and only if $k>1$.

\section{NUMERICAL EXAMPLES}

In this section, four different types numerical examples are given based the Section III. The four examples are mainly different systems: A. The regular linear singular system; B. The uniformly regular nonlinear singular system.

\section{A. The Regular Linear Singular System}

Consider the following linear singular system

$$
E \dot{x}(t)=A x(t)+B u(t)
$$

where

$$
x(t)=\left[\begin{array}{l}
x_{1}(t) \\
x_{2}(t) \\
x_{3}(t) \\
x_{4}(t)
\end{array}\right], E=\left[\begin{array}{llll}
1 & 0 & 0 & 0 \\
0 & 1 & 0 & 0 \\
0 & 0 & 1 & 0 \\
0 & 0 & 0 & 0
\end{array}\right] A=\left[\begin{array}{llll}
0 & 0 & 1 & 0 \\
1 & 0 & 0 & 0 \\
0 & 1 & 0 & 1 \\
0 & 0 & 1 & 0
\end{array}\right] B=\left[\begin{array}{l}
1 \\
1 \\
1 \\
0
\end{array}\right]
$$

On account of $\operatorname{det}(s E-A)=-s^{2}=0$ if and only if $s=0$ so the system (5) is regular. The nonsingular matrices $P$ and $Q$ can be given.

$$
P=\left[\begin{array}{cccc}
1 & 0 & 0 & -1 \\
0 & 1 & 0 & 0 \\
0 & 0 & 1 & 0 \\
0 & 0 & 0 & 1
\end{array}\right], Q=\left[\begin{array}{cccc}
1 & 0 & 0 & 0 \\
0 & 1 & 0 & 0 \\
0 & 0 & 0 & 1 \\
0 & -1 & 1 & 0
\end{array}\right]
$$

So the Kronecker form is

$$
P E Q=\left[\begin{array}{llll}
1 & 0 & 0 & 0 \\
0 & 1 & 0 & 0 \\
0 & 0 & 0 & 1 \\
0 & 0 & 0 & 0
\end{array}\right], P A Q=\left[\begin{array}{llll}
0 & 0 & 0 & 0 \\
1 & 0 & 0 & 0 \\
0 & 0 & 1 & 0 \\
0 & 0 & 0 & 1
\end{array}\right]
$$

$$
\text { and } N=\left[\begin{array}{ll}
0 & 1 \\
0 & 0
\end{array}\right], A_{1}=\left[\begin{array}{ll}
0 & 0 \\
1 & 0
\end{array}\right]
$$

Because $N \neq 0, N^{2}=0$, that is, the Kronecker indices $k=2>1$, the system (1) is impulsive based the Theorem 1 . The state response of the system (1) is given when $u(t)=\sin (t)$ and $x(0)=\left[\begin{array}{llll}0 & 0 & 0 & 0\end{array}\right]^{T}$.

As you can see from Figure 1, the system (1) is impulsive. So the result of Theorem 1 is true. 

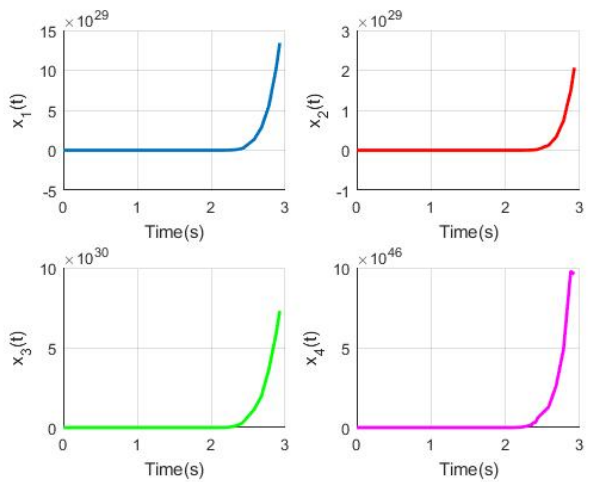

FIGURE I. THE STATE RESPONSE OF SYSTEM (5)

\section{B. The Uniformly Regular Nonlinear Singular System}

Consider the following linear singular system

$$
\left\{\begin{array}{l}
\dot{x}_{1}(t)=4 x_{1}(t)+2 x_{2}(t) \\
\dot{x}_{2}(t)=2 x_{1}(t)-x_{2}(t)-x_{2}(t) x_{3}(t)+x_{3}(t) \\
0=x_{1}(t)+2 x_{2}(t) x_{3}(t)-u(t)
\end{array}\right.
$$

The system (6) can be written as

$$
E \dot{x}(t)=\left[\begin{array}{ccc}
4 & 2 & 0 \\
2 & -1-x_{3}(t) & 1 \\
1 & 2 x_{3}(t) & 0
\end{array}\right] x(t)+\left[\begin{array}{c}
0 \\
0 \\
-1
\end{array}\right] u(t)
$$

where $x(t)=\left[\begin{array}{lll}x_{1}(t) & x_{2}(t) & x_{3}(t)\end{array}\right]^{T}, E=\left[\begin{array}{lll}1 & 0 & 0 \\ 0 & 1 & 0 \\ 0 & 0 & 0\end{array}\right]$.

The state response of the system (2) is given as Figure 2, when $u(t)=1$ and $x(0)=\left[\begin{array}{lll}1 & 1 & -0.5\end{array}\right]^{T}$, the system (2) is impulsive based on the Figure 2. Then the T-S fuzzy model of system (2) or system (3) will be given.

For the terms $x_{3}(t)$ of system (3), define $z(t)=x_{3}(t)$. Then, it has

$$
E \dot{x}(t)=\left[\begin{array}{ccc}
4 & 2 & 0 \\
2 & -1-z(t) & 1 \\
1 & 2 z(t) & 0
\end{array}\right] x(t)+\left[\begin{array}{c}
0 \\
0 \\
-1
\end{array}\right] u(t)
$$

Therefore the membership functions can be given as: $M_{11}(z(t))=1+\frac{z(t)}{2}, M_{12}(z(t))=-\frac{z(t)}{2}$.

So, the singular nonlinear system (3) can be represented by the following T-S fuzzy model.

\section{Model Rule 1:}

IF $z(t)$ is $M_{11}$, THEN $E \dot{x}(t)=A_{1} x(t)+B_{1} u(t)$;

\section{Model Rule 2:}

IF $z(t)$ is $M_{12}$, THEN $E \dot{x}(t)=A_{2}(t)+B_{2} u(t)$.

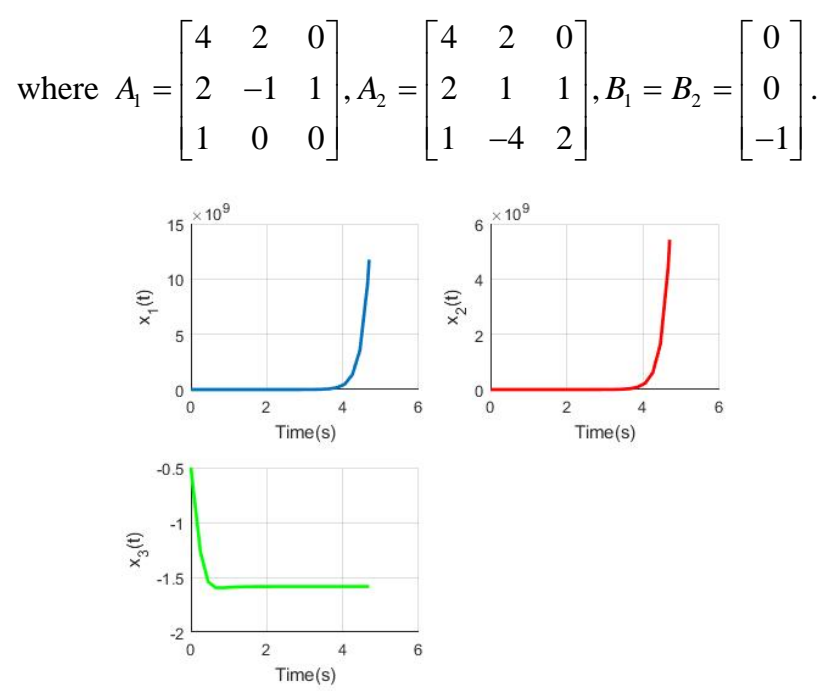

FIGURE II. THE STATE RESPONSE OF SYSTEM (6)

The system (2) or (3) can be represented by the following fuzzy model:

$$
E \dot{x}(t)=\sum_{i=1}^{2} h_{i}(z(t)) A_{i} x(t)+B_{i} u(t)
$$

where $h_{1}(z(t))=1-z(t), h_{2}(z(t))=z(t)$.

Next we analyze the regularity of the system (8).

$$
\operatorname{det}\left(s E-\sum_{i=1}^{2} h_{i}(z(t)) A_{i}\right)=(-2 z(t)-1) s+8 z(t)+2
$$

It is known that there exists $s=4$ and satisfies $\operatorname{det}\left(s E-\sum_{i=1}^{2} h_{i}(z(t)) A_{i}\right) \neq 0$ for $\forall t,(z(t) \in[-2,0]$. That is, the system (4) is uniformly regular.

Let $A=\sum_{i=1}^{2} h_{i}(z(t)) A_{i}$ and the nonsingular matrices $P$ and $Q$ can be found.

1. When $z(t) \neq-0.5$. Let $s=0$, the nonsingular $P$ and $Q$ are 


$$
P=\left[\begin{array}{ccc}
1 & 0 & \frac{-2}{2 z(t)+1} \\
-1 & -2 z(t)-1 & 4 z(t)+6 \\
0 & 0 & 1
\end{array}\right]
$$

$$
P A Q=\left[\begin{array}{lll}
1 & 0 & 0 \\
0 & 1 & 0 \\
0 & 0 & 1
\end{array}\right], P E Q=\left[\begin{array}{ccc}
0 & -1 & 0 \\
0 & 0 & -1 \\
0 & 0 & 0
\end{array}\right]
$$

So,

$$
N=\left[\begin{array}{ccc}
0 & -1 & 0 \\
0 & 0 & -1 \\
0 & 0 & 0
\end{array}\right]
$$

Because of $N \neq 0, N^{2} \neq 0$ and $N^{3}=0$, the Kronecker indices $k=3>1$, the system (6) is impulsive based the Theorem 2.

\section{CONCLUSION}

The problem of impulse analysis in the regular singular system is considered in this paper by using the Kronecker indices. Firstly, the singular system is divided into two cases based the nonlinearity. Then, the two theorems are given that the singular system is impulsive if and only if the Kronecker indices is more than one. Finally, two numerical examples have been provided to demonstrate the effectiveness of the proposed algorithms.

\section{ACKNOWLEDGMENT}

$$
N=\left[\begin{array}{cc}
0 & -1 \\
0 & 0
\end{array}\right]
$$

Because of $N \neq 0$ and $N^{2}=0$, the Kronecker indices $k=2>1$, the system (6) is impulsive based the Theorem 2 .

2. When $z(t)=-0.5$.

$$
A=\left[\begin{array}{ccc}
4 & 2 & 0 \\
2 & -0.5 & 1 \\
1 & 0 & 0
\end{array}\right]
$$

Let $s=4$, the nonsingular $P$ and $Q$ are

$$
P=\left[\begin{array}{ccc}
0 & 2 & -4 \\
-1 & 0 & 4 \\
0 & 0 & 1
\end{array}\right], Q=\left[\begin{array}{ccc}
0 & 0 & 1 \\
0 & -\frac{1}{2} & 0 \\
\frac{1}{2} & -\frac{1}{4} & 0
\end{array}\right]
$$

The Kronecker form is
The This work was supported by National Natural Science Foundation of China under Grant No.61673099.

\section{REFERENCES}

[1] S. L. Campbell, "Singular Systems of Differential Equations”, London: Pitman, 1980.

[2] S. Singh, R. W. Liu, "Existence of state equation representation of linear large-scale dynamical systems, IEEE Transactions on Circuit Theory", vol. 20, pp. 239-246, 1973.

[3] G. Zheng, D. Boutat, H. Wang "A nonlinear Luenberger-like observer for nonlinear singular systems”, Automatica, vol. 86, pp. 11-17, 2017.

[4] J. H. Li, Q. L. Zhang, X. G. Yan, and S. K. Spurgeon, "Robust stabilization of T-S fuzzy stochastic descriptor systems via integral sliding modes", IEEE Transactions on Cybernetics, 2017, doi: 10.1109/TCYB.2017.2749244.

[5] J. H. Li, Q. L. Zhang, X. G. Yan, and S. K. Spurgeon, "Integral sliding mode control for Markovian jump T-S fuzzy descriptor systems based on the super-twisting algorithm”, IET Control Theory \& Applications, vol. 11, pp. 1134-1143, 2017.

[6] G. R. Duan, “Analysis and design of descriptor linear systems”, Berlin: Springer, 2010.

[7] Q. L. Zhang, B. Y. Zhu "Analysis and control of T-S fuzzy generalized system”, Beijing: National Defense Industry Press, 2011.

[8] S. M. Potter, "Nonlinear impulse response functions", Journal of Economic Dynamics and Control, vol. 24, pp. 1425-1446, 2000.

[9] T. M. Aldybekov, "Generalized regular systems of differential equations”, Al-Farabi Kazakh National University, vol. 8, pp. 3-7, 2008.

[10] A. Bunse-Gerstner, R. Byers, V. Mehrmann, N. K.Nichols, "Feedback design for regularizing descriptor systems", Linear Algebra and it Applications, vol. 299, pp. 119-151, 1999.

[11] A. Bunse-Gerstner, V. Mehrmann, N. K.Nichols, "Regularization of descriptor systems by derivative and proportional state feedback”, Siam Journal on Matrix Analysis and Applications, vol. 13, pp. 46-67, 2006. 
[12] A. Bunse-Gerstner, V. Mehrmann, N. K.Nichols, "Regularization of descriptor systems by output feedback", IEEE Transactions on Automatic Control, vol. 39, pp. 1742-1748, 1994.

[13] R. Byers, P. Kunkel, V. Mehrmann, "Regularization of linear descriptor systems with variable coefficients", SIAM Journal on Control and Optimization, vol. 35, pp. 117-133, 1997.

[14] R. Riaza, "Singular bifurcations in higher index differential-algebraic equations”, Dynamical Systems, vol. 17, pp. 24-26, 2002.

[15] T. Berger, C. Trunk, H. Winkler, "Linear relations and the Kronecker canonical form”, Linear Algebra and Its Applications, vol. 488, pp. 1344, 2016.

[16] R. E. Beardmore, "The singularity induced bifurcation and its Kronecker normal form”, SIAM Journal on Control and Optimization, vol. 23, pp. 126-137.

[17] S. E. Marques, Y. L. Zhang, "The number of Kronecker indices of square pencils of a special kind”, Linear Algebra and Its Applications, vol. 305, pp. 15-21, 2000. 